\title{
A novel scoring system for prognostic prediction in d-galactosamine/lipopolysaccharide-induced fulminant hepatic failure BALB/c mice

\author{
Bo Feng ${ }^{\dagger 1}$, Sheng Ming Wu ${ }^{\dagger 2}$, Sa Lv ${ }^{1}$, Feng Liu ${ }^{1}$, Hong Song Chen ${ }^{1}$, Yan Gao ${ }^{1}$, \\ Fang Ting Dong*2 and Lai Wei*1
}

\begin{abstract}
Address: ${ }^{1}$ Hepatology Institute, Peking University People's Hospital, No.11 Xizhimen South Street, Beijing 100044, PR China and ${ }^{2}$ National Center of Biomedical Analysis, No.27 Taiping Road, Beijing 100039, PR China

Email: Bo Feng - xyfyfb_1@sina.com; Sheng Ming Wu - wsm@proteomics.cn; Sa Lv - lvsa@sina.com; Feng Liu - liu1116m@sina.com; Hong Song Chen - chen2999@sohu.com; Yan Gao - gaoyan6384@163.com; Fang Ting Dong* - dft@proteomics.cn; Lai Wei* - weilai@pkuph.edu.cn

* Corresponding authors †Equal contributors
\end{abstract}

Published: 30 December 2009

BMC Gastroenterology 2009, 9:99 doi:10.1 I86/147|-230X-9-99

This article is available from: http://www.biomedcentral.com/I47I-230X/9/99

(C) 2009 Feng et al; licensee BioMed Central Ltd.

This is an Open Access article distributed under the terms of the Creative Commons Attribution License (http://creativecommons.org/licenses/by/2.0), which permits unrestricted use, distribution, and reproduction in any medium, provided the original work is properly cited.
Received: 26 July 2009

Accepted: 30 December 2009

\begin{abstract}
Background: It is frequently important to identify the prognosis of fulminant hepatic failure (FHF) patients as this will influence patient management and candidacy for liver transplantation. Therefore, a novel scoring system based on metabonomics combining with multivariate logistic regression was developed to predict the prognosis of FHF mouse model.
\end{abstract}

Methods: BALB/c mice were used to construct FHF model. Parts of plasma were collected at 4, 5 , and 6-h time points after treatment, respectively, and detected using gas chromatography/timeof-flight mass spectrometry (GC/TOFMS). The acquired data were processed using partial least square discriminant analysis (PLS-DA). The metabolic markers identified were used to construct a scoring system by multivariate regression analysis.

Results: 28 mice of survival group and 28 of dead group were randomly selected and analyzed. PLS regression analysis showed that both the PLS models of $5 \mathrm{~h}$ and $6 \mathrm{~h}$ after d-galactosamine/ lipopolysaccharide treatment demonstrated good performances. Loadings plot suggested that phosphate, beta-hydroxybutyrate (HB), urea, glucose and lactate concentrations in plasma had the highest weightings on the clustering differences at the three time points. By the multivariate logistic regression analysis, the death/survival index (DSI) was constructed based on relative concentrations of HB, urea and phosphate. It provided general accurate rate of prediction of $93.3 \%$ in the independent samples.

Conclusions: The novel scoring system based on metabonomics combining with multivariate logistic regression is accurate in predicting the prognosis of FHF mouse model and may be referred in clinical practice as a more useful prognostic tool with other available information.

\section{Background}

Fulminant hepatic failure (FHF) is a syndrome characterized by an abrupt onset, hepatic encephalopathy and coagulation abnormality in the absence of preexistent liver disease [1]. The syndrome carries high morbidity and mortality. Orthotopic liver transplantation (OLT) has been increasingly the most important way for FHF, which decreased fatality rate with FHF from $85 \%$ to $35 \%$ [2]. 
Unfortunately, the application of OLT has remained low for its higher expense and less donor organs, and a high percentage of patients die before an organ is available [3]. Alternatively, with the earlier use of artificial liver supporting system (ALSS), the nonsurgical medicinal therapy was increasingly improved. Early diagnosis and prognosis identification of FHF patients is likely to result in an intervention at an earlier stage of the disease and, thus, an increased likelihood of treatment success.

In previous studies, individual compounds including lactate, phosphorus among others were proposed to predict patient outcome in FHF $[4,5]$. However, their prognostic value is debatable [6]. Application of multivariate models has been improved prediction accuracy of FHF outcome. The Clichy criteria developed in patients with hepatitis B consisted of factor V level, patient age, HBsAg and serum alfa-fetoprotein level. The King's College Hospital (KCH) criteria, which was performed based on a retrospective analysis of 585 FHF patients, have been the most widely applied [7]. However, a retrospective study about both criteria in FHF patients reported lower predictive values with the positive predictive values (PPV) at $73 \%$ to $79 \%$ and the negative predictive values (NPV) at $<50 \%[4]$. The Model for End-Stage Liver Disease (MELD) scoring system is a reliable measure of short-term mortality risk in patients with end-stage chronic liver disease. However, as a predictor of death from FHF, MELD score did not provide more information than the King's College Hospital criteria or international normalized ratio (INR) alone [8]. Therefore, better prognostic model to identify FHF patients who are likely to survive is needed.

After proposed by Nicholson in 1999, metabonomics has increasingly attained attention in recent years, applied to study various human diseases such as coronary artery disease and cancers, and provided new insights into the regulatory investigations [9-11]. One of the most important objectives of metabonomic studies is to identify novel metabolic biomarkers from biofluids in various physiological and pathological conditions. In the previous studies, we reported the dynamics of metabolic profiles in dgalactosamine/lipopolysaccharide (GalN/LPS)-treated $\mathrm{BALB} / \mathrm{c}$ mouse model, and identified 5 biomarkers including phosphate, $\beta$-hydroxybutyrate (HB), urea, glucose and lactate which may constitute a set of markers for the early diagnosis and prognosis of FHF $[12,13]$. But how to predict the prognosis based on plasma levels of these metabolic biomarkers? In this study, using metabonomic methods based on gas chromatography/time-of-flight mass spectrometry (GC/TOFMS) combined with multivariate logistic regression analysis, we aimed to derive a regression model consisted of several metabolic biomarkers for monitoring FHF, and make a basis of related investigations in clinical practice.

\section{Methods}

Materials

Male BALB/c mice (18-22 g) were purchased from the Academy of Military Medical Sciences (Beijing, China), housed in a standard animal laboratory with constant conditions of temperature, humidity, and $12 \mathrm{~h}$ light-dark cycles, and allowed free access to standard laboratory chow and water. Animal studies were carried out in accordance with the Chinese National Research Council guidelines and approved by the Subcommittee on Research Animal Care and Laboratory Animal Resources of the Peking University People's Hospital. Absolute ethanol and chloroform were obtained from Sigma (SigmaAldrich Chemical Co. Steinheim, Germany) and used in the extraction of polar metabolites. $\mathrm{N}$-methyl- $\mathrm{N}$-trimethylsilyltrifluoroacetamide (MSTFA), methoxyamine hydrochloride and pyridine were obtained from Sigma and used to derivatize pure metabolites. Ribitol was used as an internal reference. Doubly distilled water was used in the preparation of standard and sample solutions.

\section{Construction of experimental FHF model}

The FHF model was established as described previously [13]. The model group was intraperitoneally treated with $0.6 \mathrm{~g} / \mathrm{kg}$ GalN followed by $8 \mu \mathrm{g} / \mathrm{kg}$ LPS, and GalN/LPS treated mice were classed into a survival group and a dead group. Five mice of each group were randomly selected for collection of retro-orbital blood at 4,5 and $6 \mathrm{~h}$ after treatment. Additional samples obtained at $5 \mathrm{~h}$ after treatment from 23 mice of the survival group and 23 mice of the dead group were also analyzed. Blood was collected into tubes containing $10 \mu \mathrm{L}$ solution of $4 \%$ EDTA, immediately placed on ice and centrifuged at $3000 \mathrm{rpm}$ for 10 min at $4{ }^{\circ} \mathrm{C}$. Plasma samples were stored at $-80^{\circ} \mathrm{C}$ until metabonomic analysis.

\section{Assessment of liver injury}

Plasma levels of alanine aminotransferase (ALT), aspartate aminotransferase (AST) and T-bilirubin (TBIL), measured with a 7170A automatic analyzer (Hitachi, Japan), were used to assess the extent of liver injury.

\section{Plasma samples preprocessing and GCITOFMS analysis}

In this study, processing of plasma samples followed a protocol described in ref. [13]. Briefly, the thawed plasma samples were quenched by pure methanol and extracted by chloroform. The upper phase was separated and then evaporated to dryness under a stream of N2 gas. At last, the precipitation was derivatized by methoximation and trimethylsilylation.

The GC/TOFMS system consists of an HP 6890 gas chromatograph and a time-of-flight mass spectrometer (Waters Co., Milford, Massachusetts, USA). As described in previous study [13], the pretreated plasma samples 
were analyzed. Identity of GC/MS detected peaks was established by comparing their mass spectra and retention index with those available in the NIST02 library and those of commercially available reference compounds. As for some overlapping peaks, one solution to identify all the peaks in the chromatogram is to use a deconvolution software like AMDIS prior to library searching [14].

\section{Data processing}

After exported from MarkerLynx, All the collected data from the plasma samples were processed with SIMCA-P plus (version 10.0, Umetrics $A B$, Sweden) as described previously $[12,13]$, in which a range of multivariate statistical analysis were conducted. A data matrix was constructed with the sample IDs as observations and the peaks/retention times as the response variables. It was represented in a K-dimensional space (where $\mathrm{K}$ stands for the number of variables), and then projected and reduced to a few principal components that can maximize the separation between classes [15]. Here, partial least square discriminant analysis (PLS-DA) was used to process the required GC/TOFMS data. For PLS analysis, data were visualized by plotting principal component (PC) scores. In the scores, each point represents an individual sample. The plots allow the recognition of clusters of samples with similar scores. Each score plot has a loading plot associated with it, which allows in identifying the spectral regions (metabolites) responsible for the sample clustering observed [3].

To construct a regression model, multivariate regression analysis was performed based on 56 plasma samples at 5 $\mathrm{h}$ after GalN/LPS treatment using the Statistical Package for Social Scientists. The analysis was carried out with the binary response being dead or survived. This model was performed with a model group of 13 randomly selected survival samples and 13 dead samples. A validation group consisted of the remained 30 samples. The linear combination of those biomarkers identified in the metabonomic analysis was then applied to the logistic regression formula to predict the probability of FHF prognosis based on a formula score.

\section{Results}

Assessment of liver injury at $\mathbf{5} \boldsymbol{h}$ and $\mathbf{6} \boldsymbol{h}$ after treatment Compared with plasma levels of ALT, AST, and TBIL in the survival group, they were significantly increased in the dead group at $6 \mathrm{~h}$ after GalN/LPS treatment. However, plasma ALT, AST, and TBIL levels showed no significant differences between the two groups at $5 \mathrm{~h}$ time point (Table 1).

\section{Absence of ribital in plasma samples of $B A L B / c$ mice}

To confirm absence of ribitol in plasma of BALA/c mice, two samples from the same mouse were analyzed. Ribitol was added to one of them during plasma pretreatment. Figure 1 showed the peak of ribitol in the total ion current (TIC) of the sample with ribitol (upper), and there was no corresponding peak in the same retention time of TIC of another sample without ribitol (lower). Therefore, ribitol can be used as an internal standard to correct for minor variations during sample preparation and analysis.

\section{Partial least square-discriminant analysis}

PLS is a supervised statistical method and additional knowledge (Y-variables) must be included to "supervise" the independent data (X-variables). In this study, discriminant analysis was performed using PLS. The PLS diagnostic is tabulated in Table 2 after modeling. Here R2X and $\mathrm{R} 2 \mathrm{Y}$ are the fractions of $\mathrm{X}$-variation and Y-variation modeled for specific component, respectively. Q2Y is the estimate of how well the model predicts the Y-variables through a default 7 -fold cross-validation. The cumulative values of R2Y and Q2Y of the selected components close to one indicate a perfect fitting. So the diagnostic results indicated both the PLS models of $5 \mathrm{~h}$ and $6 \mathrm{~h}$ after GalN/ LPS treatment demonstrated good performances based on the acquired metabonomics data.

\section{Identification of metabolic markers for distinguishing between the survival and dead groups}

In previous studies, clustering analysis of data collected at $4 \mathrm{~h}, 5 \mathrm{~h}$ and $6 \mathrm{~h}$ was performed using PLS-DA. Score plots showed distinct clustering differences between the survival and dead groups at $5 \mathrm{~h}$ and $6 \mathrm{~h}$ after GalN/LPS treatment. Loading plots reveal the impact of metabolites on the clustering results: the closer to zero, the smaller the influence of a linear metabolite combination. All of the three loading plots in Figure 2 suggested that phosphate, $\mathrm{HB}$, urea, glucose and lactate concentrations in plasma had the highest weightings on the clustering differences although there was no clear cluster at $4 \mathrm{~h}$.

Table I: Assessment of liver injury at $5 \mathrm{~h}$ and $6 \mathrm{~h}$ after GaIN/LPS treatment

\begin{tabular}{ccccc}
\hline & Parameters & Survival group & Dead group & $P$ \\
\hline \multirow{2}{*}{$5 \mathrm{~h}$} & ALT (U/L) & $698.52 \pm 256.37$ & $722.34 \pm 242.87$ & $4.13 \mathrm{E}-01$ \\
& AST $(\mathrm{U} / \mathrm{L})$ & $712.45 \pm 301.23$ & $745.21 \pm 297.38$ & $5.42 \mathrm{E}-02$ \\
\multirow{2}{*}{$6 \mathrm{~h}$} & TBIL $(\mu \mathrm{mol} / \mathrm{L})$ & $3.12 \pm 0.96$ & $3.23 \pm 1.13$ & $1.75 \mathrm{E}-01$ \\
& ALT $(\mathrm{U} / \mathrm{L})$ & $762.12 \pm 212.5 \mathrm{I}$ & $1967.34 \pm 1032.78$ & $2.15 \mathrm{E}-03$ \\
& AST $(\mathrm{U} / \mathrm{L})$ & $801.92 \pm 246.73$ & $1874.54 \pm 952.65$ & $4.02 \mathrm{E}-04$ \\
& TBIL $(\mu \mathrm{mol} / \mathrm{L})$ & $3.37 \pm 1.02$ & $5.12 \pm 1.31$ & $3.23 \mathrm{E}-03$ \\
\hline
\end{tabular}




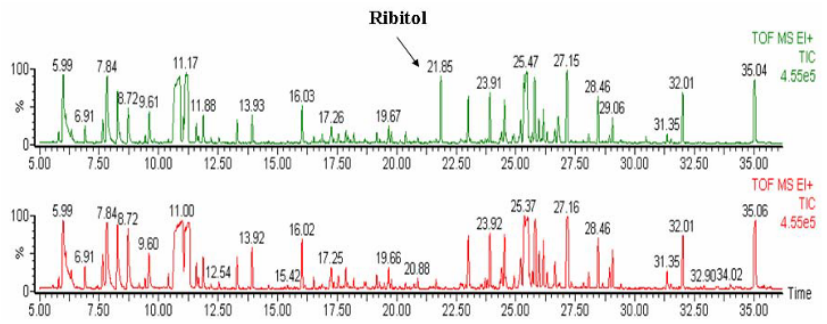

Figure I

Absence of ribital in plasma samples of BALB/c mice. The peak of ribitol was showed in TIC of the sample with ribitol (upper), and there was no corresponding peak in the same retention time of TIC of another sample without ribitol (lower).

\section{Regression analysis of data at $5 \mathrm{~h}$ point}

Backward stepwise, which is one of three main approaches to automating the selection of explanatory variables for inclusion in a regression model, was used in the multivariate logistic regression analysis. In backward selection, all variables are included in the initial model, and then removed sequentially until a final model is produced. After 3 steps, lactate and glucose were removed, and other variables ( $\mathrm{HB}$, urea and phosphate) were used to calculate a nomogram. The derived nomogram, the death/survival index (DSI) was:

$$
0.027 \mathrm{HB}+0.031 \mathrm{UREA}-0.035 \mathrm{PHOS}-5.732 .
$$

Probability of survival as compared to death was calibrated from the logistic regression analysis using the equation as follows:

$$
P=e^{D S I} /\left(1+e^{D S I}\right) .
$$

Table 2: Modeling diagnostic of the metabolic data derived from plasma samples of various time points after GaIN/LPS

\begin{tabular}{|c|c|c|c|c|}
\hline \multirow[t]{2}{*}{ PLS model } & \multirow[t]{2}{*}{ Components } & \multicolumn{3}{|c|}{ Modeling diagnostic } \\
\hline & & $\mathbf{R} 2 \mathbf{X}$ & $\mathbf{R} 2 \mathbf{Y}$ & Q2Y \\
\hline \multirow[t]{3}{*}{$4 \mathrm{~h}$} & 1 & 0.186 & 0.288 & 0.225 \\
\hline & 2 & 0.256 & 0.412 & 0.368 \\
\hline & 3 & 0.335 & 0.556 & 0.406 \\
\hline \multirow[t]{3}{*}{$5 \mathrm{~h}$} & I & 0.351 & 0.742 & 0.701 \\
\hline & 2 & $0.47 \mid$ & 0.876 & 0.812 \\
\hline & 3 & 0.504 & 0.915 & 0.857 \\
\hline \multirow[t]{3}{*}{$6 \mathrm{~h}$} & I & 0.367 & 0.781 & 0.666 \\
\hline & 2 & 0.433 & 0.896 & 0.817 \\
\hline & 3 & 0.493 & 0.941 & 0.862 \\
\hline
\end{tabular}
treatment

Note. I, 2, or 3 indicates quantities of components contributed to cumulative values. $R 2 X$ is the fraction of $X$-variation; $R 2 Y$ is the fraction of $Y$-variation; $Q 2 Y$ is the cumulated cross-validation for R2Y. R2X, R2Y and Q2Y are all ranged from 0 to I.
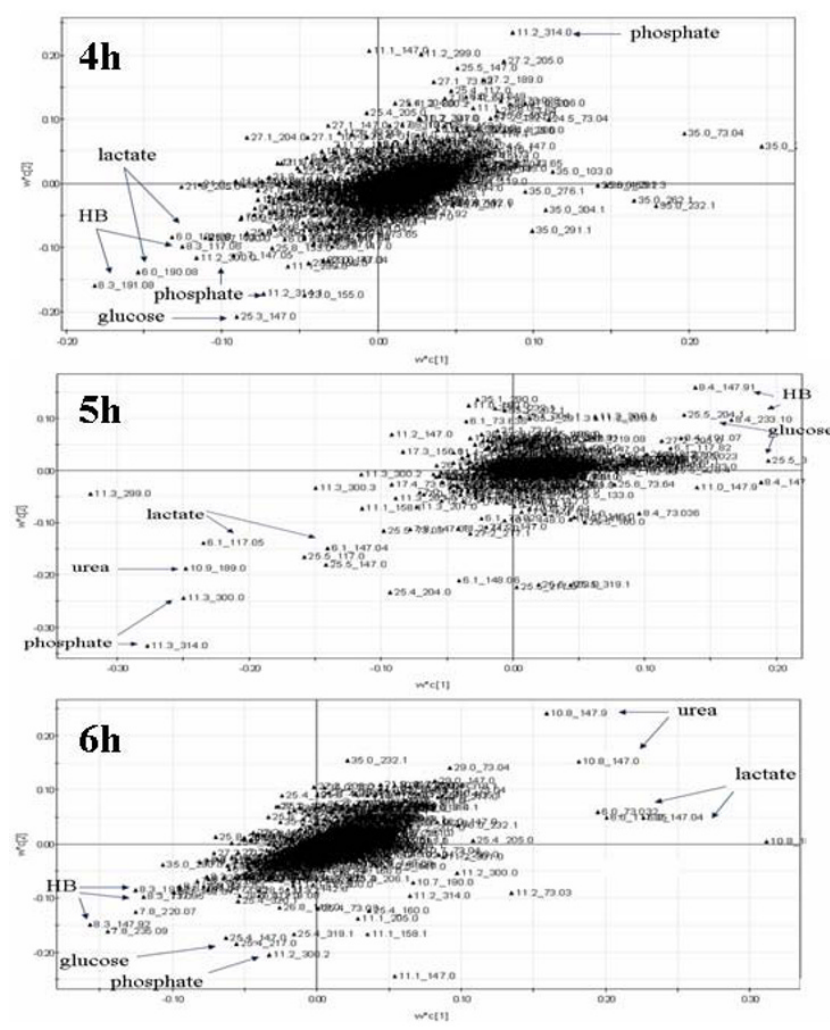

Figure 2

Identification of metabolic markers for distinguishing between survival and dead groups. Phosphate, HB, urea, glucose and lactate concentrations in plasma had the highest weightings on the clustering differences at $4 \mathrm{~h}, 5 \mathrm{~h}$ and $6 \mathrm{~h}$ after GalN/LPS treatment, although there was no clear cluster at $4 \mathrm{~h}$.

The resulting value was defined as the DSI, with DSI $<0$ incrementally favored a prognosis of "death", while DSI > 0 corresponded to a higher likelihood of a prognosis of "survival".

\section{Validation of the death/survival index (DSI)}

We further validated and calibrated the DSI by comparing the predicted probability of FHF prognosis based on DSI with the validation group. Based on the cut-off value of DSI as above, one of 15 survival samples and one of 15 dead samples were removed, and general accurate rate of prediction was $93.3 \%$. Figure 3 shows predicted probability of survival based on DSI. DSI of $>0.65$ or $<-0.65$ provided a relatively clear diagnosis, corresponding to a $93.3 \%$ or $6.7 \%$ probability of survival.

\section{Discussion}

It is well known that various biological factors affect the metabolic composition of biological fluids [15]. They are intrinsic (e.g. age, gender) and extrinsic (e.g. diet, smok- 
ing, drugs). External variation can be minimized in studies with laboratory animals, whereas in humans these factors are more difficult to control. Alternatively, because of higher fees, more explicit method and larger data, it is difficult to directly apply GC/TOFMS for clinical practice now. However, it is easy and applicable to construct a scoring system for early identification of diseases' prognosis based on metabolic biomarkers.

The rapidly developing field of metabonomics offered a new method to identify biomarkers. Nuclear magnetic resonance (NMR) and mass spectrometry (MS) are the most frequently employed methods of detection in the analysis of the metabolome $[16,17]$. NMR provides a rapid, non-destructive, high-throughput method that requires little or no sample preparation, and however, its equipment cost is much higher and its sensitivity is lower limited than MS. The most advantages of MS are its high sensitivity, and high-throughput. Furthermore, the combination of separation techniques with MS tremendously expands the capability of the chemical analysis of highly complex biological samples. Among the tandem techniques investigated, the coupling of capillary gas chromatography to MS (GC/MS) proved to be a potentially useful method based on its high sensitivity, peak resolution and reproducibility. The availability of GC/MS electron impact (EI) spectral libraries further facilitates the identification of diagnostic biomarkers and aids the subsequent mechanistic elucidation of the biological or pathological variations [14].

In the clinical arena, it is frequently important to identify the prognosis of FHF patients (survival or death) as this will influence patient management and candidacy for liver transplantation. Despite a number of novel and sometimes expensive biomarkers that have been devel-

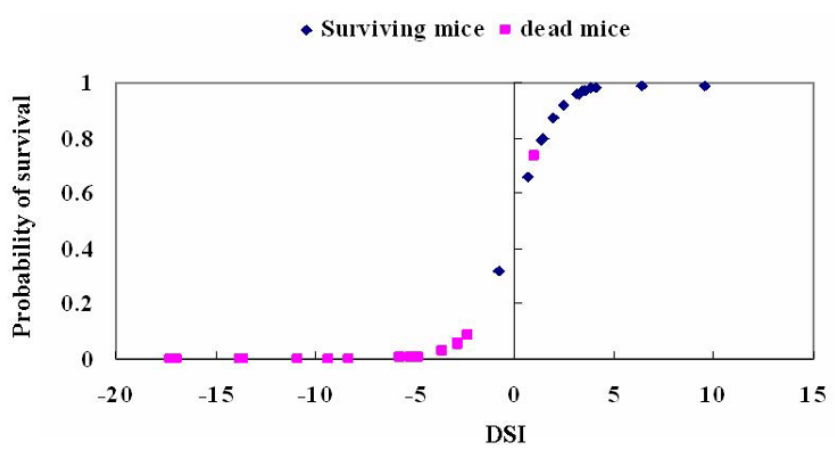

Figure 3

Predicted probability of survival based on the death/ survival index (DSI). DSI of $>0.65$ or $<-0.65$ provided a relatively clear diagnosis, corresponding to a $93.3 \%$ or $6.7 \%$ probability of survival. Squares represent dead mice, and diamonds represent surviving mice. oped and evaluated, most of these have not been reproducibly demonstrated to perform more effectively than those traditional and inexpensive laboratory values $[4,8]$. GC/TOFMS of biofluids and tissues coupled with appropriate statistical methods offer a novel and robust approach for identifying individual metabolites or combinations of metabolites that may serve as pathological biomarkers. This results in the generation of an endogenous metabolic profile that has the potential to reveal characteristics indicative of disease status.

To construct a model for early prediction of FHF prognosis, in previous studies we investigated the dynamics of metabolic intermediates and metabolic profiles using a GalN/LPS-treated BALB/c mouse model of FHF prior to clinical FHF patients $[12,13]$. In this study, PLS-DA was used to obtain a list of potential biomarkers which are statistically significant and which separate one class from another. The PLS diagnostic tabulated in Table 2 contained three factors, showing the performance statistics of $\mathrm{R} 2 \mathrm{X}, \mathrm{R} 2 \mathrm{Y}$ and $\mathrm{Q} 2 \mathrm{Y}$. R2X is the cumulative modeled variation in the GC/TOFMS response variables, $\mathrm{X}$; R2Y is the cumulative modeled variation in the observation variables, $\mathrm{Y}$; and Q2Y is the cumulative predicted variation in $\mathrm{Y}$, according to cross-validation. The ranges of these parameters are $0-100 \%$, where $100 \%$ indicates a perfect fit [18]. So the diagnostic results indicated both the PLS models of $5 \mathrm{~h}$ and $6 \mathrm{~h}$ after GalN/LPS treatment demonstrated good performances based on the acquired metabonomics data. That means distinct clustering (survival and death) was obtained at $5 \mathrm{~h}$ and $6 \mathrm{~h}$ time points. To construct an early prediction model, we selected $5 \mathrm{~h}$ time point when there was no significant difference in plasma levels of ALT, AST, and TBIL between the two groups. Loadings plot represented the impact of metabolites on the clustering results: the closer to zero, the smaller the influence of a linear metabolite combination. Phosphate, $\mathrm{HB}$, urea, glucose and lactate concentrations in plasma had the highest weightings on the clustering differences at the three time points, although there was no clear cluster at $4 \mathrm{~h}$ after treatment. It was suggested that a combination of phosphate, $\mathrm{HB}$, urea, glucose and lactate concentrations in the plasma could potentially be used to separate the survival and dead groups earlier.

Based on the set of potential metabolic biomarkers including phosphate, HB, urea, glucose and lactate identified using GC/TOFMS combined with multivariate statistics, we attempted to form a scoring system to early identify FHF outcomes. By the multivariate logistic regression analysis, lactate and glucose were removed, and then the death/survival index (DSI) was constructed based on relative concentrations of other variables $(\mathrm{HB}$, urea and phosphate) and validated well in the samples from independent validation group. The DSI maintains important 
and unique methodological aspects that enhance its utility as compared to other studies designed to predict FHF prognosis. The use of logistic regression facilitated appropriate weighting of the parameters that comprise the DSI. Alternatively, the DSI is a continuous variable, we can identify the probability of FHF prognosis (survival or death) based on the magnitude and dynamic change of the DSI.

\section{Conclusions}

We have developed a novel scoring system that is highly accurate in predicting the prognosis of FHF mouse model. The methodological aspects based on metabonomic technologies combining with multivariate logistic regression were constructed and may be referred in clinical practice. As a useful prognostic tool, it may be complemented with other available information such as traditional laboratory values and clinical parameters.

\section{Potential limitations}

Various biological factors affect the metabolic composition of biological fluids, and these factors might be involved in humans to different extend. Although our previous pilot result showed that changes of plasma phosphate, $\mathrm{HB}$, urea, glucose and lactate levels in FHF patients were consistent with those of FHF mouse model, it is unknown whether the scoring system based on the five metabolites would be applicable to predicting the prognosis FHF seen in clinical practice. In addition, etiology of FHF patients is complex, which can influence metabonomics of body fluids. All of these need a further investigation.

\section{Abbreviations}

FHF: fulminant hepatic failure; GalN: d-galactosamine; LPS: lipopolysaccharide; HB: $\beta$-hydroxybutyrate; GC/ TOFMS: gas chromatography/time-of-flight mass spectrometry; PLS-DA: partial least square discriminant analysis; TIC: total ion current; DSI: death/survival index

\section{Competing interests}

The authors declare that they have no competing interests.

\section{Authors' contributions}

BF contributed to design, experimental process, data acquisition, statistical analysis and drafted the manuscript. SMW contributed to experimental process, data acquisition, statistical analysis and drafted the manuscript. SL participated in experimental process and analysis. FL participated in study planning, data acquisition and statistical analysis. HSC contributed to data analysis and to the manuscript. YG participated to planning and statistical analysis. FTD contributed to study design, data analysis and drafting of the manuscript. LW participated in design, funding analysis and manuscript drafting. All authors read and approved the final manuscript.

\section{Acknowledgements}

The authors gratefully acknowledge funding support by the Major State Basic Research Development Program of China (grant numbers 2005CB522902 and 2007CB5 I2906), the National High Technology Research and Development Program of China (grant number 2006AA02A4 I0), the National Nature Science Foundation of China (grant number 30571639), the National Science and Technology Key Project during the II th Five-Year Plan Period (grant numbers 2008ZX 10002-0I 2 and 2008ZX10002-013) and Key Clinical Research Program of Ministry of Health.

\section{References}

I. Shakil AO, Mazariegos GV, Kramer DJ: Fulminant hepatic failure. Surg Clin North Am 1999, 79:77-108.

2. Polson J, Lee WM: AASLD position paper: the management of acute liver failure. Hepatology 2005, 4 I: I I79-1 I 97.

3. Barba I, Chatauret N, Garcia-Dorado D, Cordoba J: A I H nuclear magnetic resonance-based metabonomic approach for grading hepatic encephalopathy and monitoring the effects of therapeutic hypothermia in rats. Liver Int 2008, 28: I I I I- I I 48.

4. Chung PY, Sitrin MD, Te HS: Serum phosphorus levels predict clinical outcome in fulminant hepatic failure. Liver Transpl 2003, 9:248-253.

5. MacQuillan GC, Seyam MS, Nightingale P, Neuberger JM, Murphy N: Blood lactate but not serum phosphate levels can predict patient outcome in fulminant hepatic failure. Liver Transpl 2005, I I:1073-1079.

6. Shakil AO: Predicting the outcome of fulminant hepatic failure. Liver Transpl 2005, I I:1028-1030.

7. Shakil AO, Kramer D, Mazariegos GV, Fung JJ, Rakela J: Acute liver failure: clinical features, outcome analysis, and applicability of prognostic criteria. Liver Transp/ 2000, 6:163-169.

8. Schmidt LE, Larsen FS: MELD score as a predictor of liver failure and death in patients with acetaminophen-induced liver injury. Hepatology 2007, 45:789-796.

9. Barba I, de Leon Gustavo, Martin E, Cuevas A, Aquade S, CandellRiera J, Barrabes JA, Garcia-Dorado D: Nuclear magnetic resonance-based metabolomics predicts exercise-induced ischemia in patients with suspected coronary artery disease. Magn Reson Med 2008, 60:27-32.

10. Serkova NJ, Gamito EJ, Jones RH, O'Donnell C, Brown JL, Green S, Sullivan H, Hedlund T, Crawford ED: The metabolites citrate, myo-inositol, and spermine are potential age-independent markers of prostate cancer in human expressed prostatic secretions. Prostate 2008, 68:620-628.

II. Gottschalk M, Ivanova G, Collins DM, Eustace A, O'Connor R, Brougham DF: Metabolomic studies of human lung carcinoma cell lines using in vitro IH NMR of whole cells and cellular extracts. NMR Biomed 2008, $21: 809-819$.

12. Feng B, Wu S, Lv S, Liu F, Chen H, Yan X, Li Y, Dong F, Wei L: Metabolic profiling analysis of a d-galactosamine/lipopolysaccharide-induced mouse model of fulminant hepatic failure. J Proteome Res 2007, 6:2161-2167.

13. Feng B, Wu S, Lv S, Fang J, Liu F, Li Y, Gao Y, Yan X, Dong F, Wei L: Dynamic metabonomic analysis of $B A L B / c$ mice with different outcomes after d-galactosamine/lipopolysaccharideinduced fulminant hepatic failure. Liver Transpl 2008, 14:1620-1631.

14. Pasikanti KK, Ho PC, Chan ECY: Development and validation of a gas chromatography/mass spectrometry metabonomic platform for the global profiling of urinary metabolites. Rapid Commun. Mass Spectrom 2008, 22:2984-2992.

15. Psihogios NG, Gazi IF, Elisaf MS, Seferiadis KI, Bairaktari ET: Genderrelated and age-related urinalysis of healthy subjects by NMR-based metabonomics. NMR Biomed 2008, 21:195-207.

16. Bedair M, Sumner LW: Current and emerging mass spectrometry technologies for metabolomics. Trends Analyt Chem 2008, 27:238-250. 
17. Li X, Cristina LQ: Advances in separation science applied to metabonomics. Electrophoresis 2008, 29:3724-3736.

18. Lu Y, A J, Wang G, Hao H, Huang Q, Yan B, Zha W, Gu S, Ren H, Zhang Y, Fan X, Zhang M, Hao K: Gas chromatography/time-offlight mass spectrometry based metabonomic approach to differentiating hypertension- and age-related metabolic variation in spontaneously hypertensive rats. Rapid Commun Mass Spectrom 2008, 22:2882-2888.

\section{Pre-publication history}

The pre-publication history for this paper can be accessed here:

http://www.biomedcentral.com/1471-230X/9/99/pre pub

Publish with Bio Med Central and every scientist can read your work free of charge

"BioMed Central will be the most significant development for disseminating the results of biomedical research in our lifetime. "

Sir Paul Nurse, Cancer Research UK

Your research papers will be:

- available free of charge to the entire biomedical community

- peer reviewed and published immediately upon acceptance

- cited in PubMed and archived on PubMed Central

- yours - you keep the copyright

Submit your manuscript here:

http://www.biomedcentral.com/info/publishing_adv.asp 